\title{
Epidemiological Determinants of Acute Respiratory Syndrome Coronavirus-2 Disease Pandemic and The Role of the Bacille-Calmette-Guerin Vaccine in Reducing Morbidity and Mortality
}

\author{
Bhoj R. Singh ${ }^{1 *}(\mathbb{D})$, Richa Gandharva ${ }^{2}$ (D) R. Karthikeyan ${ }^{1}(D)$, Shiv Varan Singh ${ }^{3}$ (D), \\ Akanksha Yadav ${ }^{1}$ (D), Vinodh Kumar O.R. ${ }^{1}$ (D), Dharmendra K. Sinha ${ }^{1}$ (D), Varsha \\ Jayakumar ${ }^{1} \mathbb{D}$, Kuldeep Dhama ${ }^{4} \mathbb{D}$, Dharmender Kumar $^{5}(\mathbb{D}$ and Sumedha \\ Gandharava $^{6}$ (i) \\ ${ }^{1}$ Division of Epidemiology, ICAR-Indian Veterinary Research Institute, Izatnagar-243122, India. \\ ${ }^{2}$ Legal Advisor on International Trade and Investment Laws, 23A Vaibhav, Suncity Vistaar, Bareilly, 243122, India. \\ ${ }^{3}$ Division of Bacteriology and Mycology, ICAR-Indian Veterinary Research Institute, Izatnagar-243122, India. \\ ${ }^{4}$ Avian Diseases, Division of Pathology, ICAR-Indian Veterinary Research Institute, Izatnagar-243122, India. \\ ${ }^{5}$ Department of Biotechnology, DCR University of Science \& Technology, Murthal-131039 Sonepat Haryana India. \\ ${ }^{6}$ Department of Engineering, Boise State University, Idaho, USA.
}

*Correspondence: brs1762@gmail.com; +91-8449033222

(Received: April 15, 2020; accepted: April 21, 2020)

Citation: Singh BR, Gandharva R, Karthikeyan R, et al. Epidemiological Determinants of Acute Respiratory Syndrome Coronavirus-2 Disease Pandemic and The Role of the Bacille-Calmette-Guerin Vaccine in Reducing Morbidity and Mortality. J Pure App/ Microbiol. 2020;14(suppl 1):1007-1016. doi: 10.22207/JPAM.14.SPL1.39

C The Author(s) 2020. Open Access. This article is distributed under the terms of the Creative Commons Attribution 4.0 International License which permits unrestricted use, sharing, distribution, and reproduction in any medium, provided you give appropriate credit to the original author(s) and the source, provide a link to the Creative Commons license, and indicate if changes were made. 


\begin{abstract}
This study analyzed the determinants of morbidity, mortality, and case fatality rate (CFR) of the ongoing pandemic of severe acute respiratory syndrome coronavirus-2 disease 2019 (COVID-19). Data for 210 countries and territories available in public domains were analyzed in relation to mandatory vaccination with Bacille-Calmette-Guerin (BCG), population density, median age of the country population, health care expenditure per capita, life expectancy at birth, healthy life expectancy, literacy rate, per capita gross domestic production adjusted to purchasing power (PPP), burden of tuberculosis (TB), acquired immunodeficiency disease caused by human immunodeficiency virus (HIV-AIDS), malaria, cardiovascular disease (CVD), neoplasm, diabetes, deaths due to energy-protein (food) deficiency (EPD), and per capita government spending on safe water and sanitation. Mandatory BCG vaccination showed a highly significant $(p<0.0001)$ negative correlation with COVID-19 morbidity $(r=-0.62)$ and mortality $(r=$ $-0.58)$ rates, but no significant correlation with CFR. The median age of the nation showed a significant $(p<0.0001)$ positive correlation with COVID-19 morbidity $(r=0.40)$ and mortality $(r=0.34)$ rates, but no significant correlation with CFR. The pandemic resulted in higher morbidity $(r=0.47, p<0.0001)$ and mortality $(r=0.25, p=0.01)$ rates in countries with a higher PPP than in those with a lower PPP. COVID-19 CFR and morbidity and mortality rates showed no significant correlation with population density, the burden of malaria or diabetes, or the level of spending on safe water and sanitation. Only the burden of TB showed a positive correlation with CFR $(r=0.17, p=0.05)$. However, COVID-19 morbidity showed a significant $(p \leq 0.05)$ negative correlation with the burden of TB, HIV-AIDS, CVD, and EPD. Mortality and morbidity in COVID-19 patients showed a positive correlation with per capita health expenditure, life expectancy, the burden of neoplasia, and PPP.
\end{abstract}

Keywords: COVID-19, SARS-CoV-2, Epidemiology, Effectors, PPP, BCG vaccine, TB, CVD, Poverty

\section{INTRODUCTION}

Novel Coronavirus or severe acute respiratory syndrome Coronavirus-2 (SARS-CoV-2) infection causes the Coronavirus disease known as COVID-19, which is presently a devastating pandemic. It has spread throughout most of the world (210 countries and territories) and has affected more than 2.52 million people. With a high case fatality rate (CFR, 21\%) COVID-19 has killed more than 174,000 people $^{1}$. Besides morbidity and mortality, the pandemic may lead to a global economic depression, which will be evident in the coming months and years, leaving millions of people jobless, homeless, and foodless. Despite the fact that lockdown and social distancing in response to such contagious diseases have negative socioeconomic effects ${ }^{2,3}$, almost half of the world is in lockdown today from the widespread fear of COVID-19. An understanding of epidemiology and the determinants of disease spread, morbidity, and mortality, in addition to socioeconomic cost-benefit analyses are prerequisites for overcoming this problem. Although monkeys have been shown to be susceptible ${ }^{4}$ and some cats, dogs, and tigers tested positive for COVID-19 infection, ${ }^{5-7}$, the disease is mainly spreading through human-to-human contact. However, the epidemiology of COVID-19 remains unclear with respect to its origin and the reservoir and intermediate hosts (if any). The aim of this study was to analyze the association of different socioeconomic and population demographic factors with morbidity, mortality, CFR, and percent positivity among people tested for COVID-19, using data available in the public domain.

\section{Data Sources}

Data from different countries related to the total number of COVID-19 tests, the positivity rate in tested samples, morbidity, mortality, CFR, and population density were retrieved from Worldometer ${ }^{8}$. The gross domestic product per capita (using the purchase power parity [PPP] metric) $)^{9,10}$ and data pertaining to the use of Bacille-Calmette-Guerin (BCG) vaccination ${ }^{11,12}$; literacy rate; median age ${ }^{13}$; heath care expenditure per capita; life expectancy at birth; healthy life expectancy; the burden of tuberculosis (TB), acquired immunodeficiency disease caused by human immunodeficiency virus (HIV-AIDS), malaria, cardiovascular disease (CVD), neoplasm, and diabetes; deaths due to energy-protein (food) deficiency (EPD); and per capita government 
spending on safe water and sanitation ${ }^{14,15}$ were downloaded from various websites in the public domain.

These data were entered into a Microsoft Excel2007 spreadsheet for tabulation, analysis (Chi squared test and calculations of correlation coefficients and odds ratios [ORs]), ranking, and other studies. The significance of each correlation was determined using standard reference tables.

\section{RESULTS AND DISCUSSION}

Factors showing a significant correlation with morbidity, mortality, CFR, and percent positivity for COVID-19

The factors showing a significant positive or negative correlation with COVID-19 mortality (Fig. 1) and morbidity (Fig. 2) rates, CFR, and percent positivity among tested individuals are listed in Table 1 \& 2, which show odds ratios and statistical significance.

BCG vaccination, TB burden, and COVID-19

In most developed countries, BCG vaccination ceased many years ago, after the countries were almost free of TB or achieved a low TB burden (less than 100 cases per million people $)^{14,16}$. The BCG vaccine is controversial due to its questionable efficacy in protecting from Mycobacterium tuberculosis infection ${ }^{11,12}$. The analysis of COVID-19 data from 190 countries with known BCG vaccination status revealed that morbidity $(r=-0.62, p<0.0001)$ and mortality $(r$ $=-0.58, p<0.0001)$ rates and percent positivity among individuals tested for SARS-CoV-2 infection ( $r=-0.25, p=0.01$ ) showed significant negative correlations with the mandatory use of the BCG vaccine (Table 2 ). This observation indicated the protective efficacy of childhood BCG vaccination in protecting from COVID-19 morbidity and mortality. A plausible reason may be the induction of nonspecific and cross-protective immunity by BCG vaccination ${ }^{17,18}$, even against viral infections.

Up to April 15, 2020, COVID-19 morbidity (Fig. 3) and mortality (Fig. 4) rates were lower in countries with mandatory BCG vaccination than in those with optional or no BCG vaccination. The odds of having $>500$ COVID-19 cases per million people was 133 times higher in countries with non-mandatory BCG vaccination than in those with mandatory BCG vaccination $(99 \% \mathrm{Cl}, 17.5-1011.37$; Table 2). The odds of having $>50$ COVID-19-related deaths per million people was 42.96 times higher in countries without mandatory BCG vaccination

Table 1. Modulators of morbidity, mortality, case fatality rates, and percent positivity of COVID-19 in the tested population

Attributes of Negative Modulators Positive Modulators

COVID-19

Morbidity per Mandatory BCG vaccination ( $r=0.62$, Higher median age $(r=0.40, p<0.001)$, per capita million people $p<0.001)$, burden of tuberculosis $(r=-$ spending on the health system $(r=0.47, p<0.001)$, $0.20, p=0.01)$, HIV-AIDS ( $r=-0.18 ; \quad$ higher life expectancy $(r=0.36, p<0.001)$, healthy life $0.05)$, cardiovascular disorders $(r=-\quad$ expectancy $(r=0.45, p<0.001)$, higher literacy rates $(r=$ $0.36, p<0.001)$, and energy-protein $\quad 0.21, p<0.01)$, incidence of neoplasia $(r=0.471$, deficiency $(r=-0.19, p<0.05) \quad p<0.001)$, \& higher per capita income $(r=0.47, p<0.001)$

Mortality per Mandatory BCG vaccination $(r=-0.58$, Higher median age $(r=0.34, p<0.001)$, per capita million people $p<0.001)$, burden of tuberculosis $(r=-$ spending on the health system $(r=0.25, p<0.01)$, $0.17, p=0.05)$, and energy-protein higher life expectancy $(r=0.30, p<0.001)$, healthy life deficiency $(r=-0.19, p<0.05) \quad$ expectancy $(r=0.45, p<0.001)$, higher per capita income $(r=0.25, p<0.01)$, incidence of neoplasm $(r=$ $0.39, p<0.001)$

Case fatality None

rate Per capita spending on the health system $(r=0.16$, $p<0.05)$ and burden of tuberculosis $(r=0.17, p<0.05)$

Percent Mandatory BCG vaccination ( $r=-0.25$, Higher per capita spending on the health system $(r=$ positivity among $p<0.01)$ and spending on safe water $0.18, p<0.05)$ and higher life expectancy $(r=0.19$, those tested and sanitation $(r=-0.17, p=0.05) \quad p<0.05)$

BCG, Bacille-Calmette-Guerin 
than in those with mandatory BCG vaccination (99\% Cl, 9.62-191.85). These observations are in concurrence to earlier epidemiological analytical reports ${ }^{19,20}$, indicating a strong positive protective effect of BCG vaccination against COVID-19 morbidity and mortality. A recent World Health Organization report ${ }^{21}$, with no epidemiological analysis, claimed that there is no evidence for protection against COVID-19 with the use of the BCG vaccine. Based on epidemiological evidence suggesting low COVID-19 morbidity and mortality rates in nations with mandatory BCG vaccination, Australia and the Netherlands have initiated clinical trials ${ }^{22,23}$.

The burden of TB was also negatively correlated $(p=0.05)$ with COVID-19 mortality $(r$ $=-0.17)$ and morbidity $(r=-0.19)$ rates. This may be owing to the association of Mycobacterium spp. with the non-specific induction of immunity against viral diseases ${ }^{17,18}$. Although COVID-19 morbidity (OR 24.46; $99 \% \mathrm{Cl}, 1.72-347.8)$ and mortality (OR $10.83 ; 95 \% \mathrm{Cl}, 1.57-92.2$ ) rates were significantly higher in countries with a low TB burden ( $<100$ deaths/million/year) than in countries with a high TB burden, COVID-19 CFR was positively correlated with TB burden $(r=0.17$, $p=0.05)$. This observation indicated that $\mathrm{TB}$, which may result in injured or stressed lungs, may make a patient more prone to the respiratory failures seen with COVID-19. Underlying disorders that cause respiratory distress have been identified as important co-morbidities leading to lethality in COVID-19 patients ${ }^{24}$. However, further studies are required to test this hypothesis.

The median age of the population and COVID-19 morbidity, mortality, and CFR

Neither CFR nor percent positivity among individuals tested for COVID-19 showed a significant correlation with the median age of the population. COVID-19-related morbidity (Fig. 3) and mortality (Fig. 4) rates were higher in countries with a higher median age ( $>30$ years) than in those with a lower median age $(\leq 30$ years). However, countries with a high ( $>40$ years) median age had a greater chance of having higher morbidity (OR 11.2 ; $99 \% \mathrm{Cl}, 2.68-47.9$ ) and mortality (OR $8.18 ; 99 \% \mathrm{Cl}, 1.57-42.49)$ rates than those with a low ( $\leq 30$ years) or medium
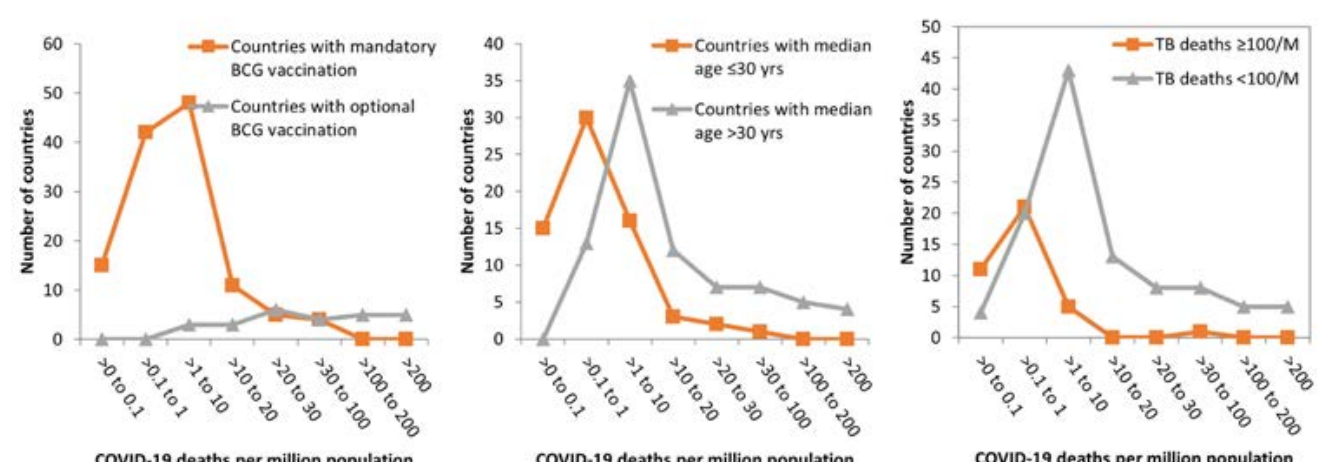

COVID-19 deaths per million population

COVID-19 deaths per million population
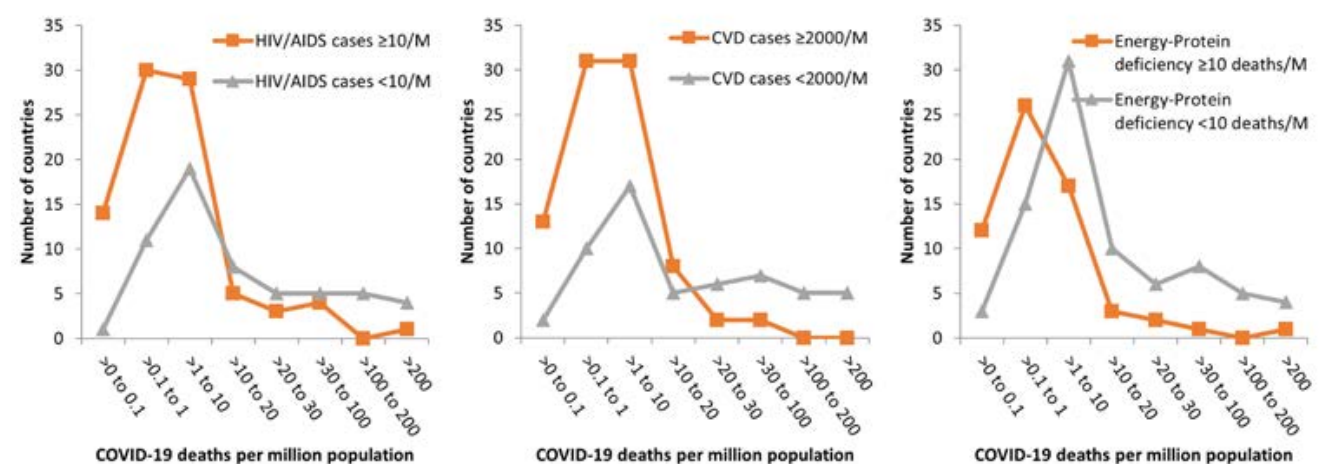

Fig. 1. Determinants of COVID-19 mortality (per million population) patterns 
(30-40 years) median age (Table 2). The median age of the population showed a strong positive correlation with COVID-19 mortality $(r=0.34$, $p=0.0001)$ and morbidity $(r=0.40, p=0.0001)$ rates. High COVID-19 morbidity and mortality rates have been reported to be associated with other co-morbidities of old age ${ }^{24}$. However, the positive correlation between high median age and morbidity $(r=0.44, p=0.0001)$ and mortality $(r=$ $0.35, p=0.0001$ ) rates in countries with a greater healthy life expectancy, indicated that the role of co-morbidities may be modulated by some currently unknown confounders of COVID-19 and median age associations.

After including the effects of both median age and BCG vaccination in the analysis, mandatory BCG vaccination was found to be an important negative modulator of SARS-CoV-2 infection, irrespective of median age (Table 2).

Disease burden and COVID-19 morbidity, mortality, and CFR

Besides TB, as discussed above, the burden of HIV-AIDS and CVD and EPD death rates also showed significant negative correlations with either COVID-19 morbidity, mortality, or both (Table 1). However, these factors did not show a significant correlation with COVID-19 CFR. Neither the prevalence of malaria nor diabetes showed a significant correlation with COVID-19 CFR, morbidity, or mortality. However, in earlier case-to-case analyses, diabetics have been shown to be more prone to COVID-19-related mortality ${ }^{25}$. A higher burden of neoplasia was strongly associated with increased COVID-19 morbidity $(r=0.47, p=0.0001)$ and mortality $(r=0.39, p=$ $0.0001)$ rates. Neoplasia has been reported to be an important co-morbidity associated with worse outcomes of SARS-CoV-2 infection in different countries ${ }^{24-27}$. Though several co-morbidities have been shown ${ }^{24-27}$ to modulate the outcome of COVID-19, further studies and targeted data analysis are needed to better understand the relationship between these diseases.

COVID-19 and population density

Population density showed no significant correlation with COVID-19 CFR, morbidity, or mortality. This is contrary to the popular belief that contagious diseases spread faster in densely populated countries ${ }^{28}$ because of the greater chance of interpersonal contact. This contradiction

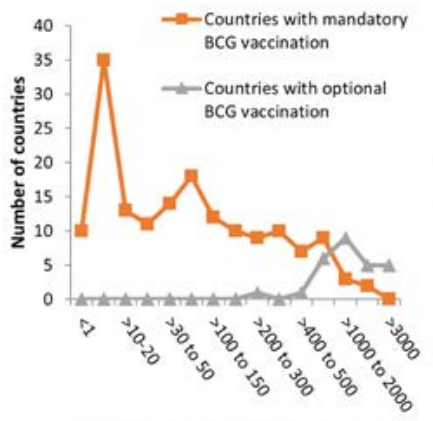

COVID-19 cases per million population

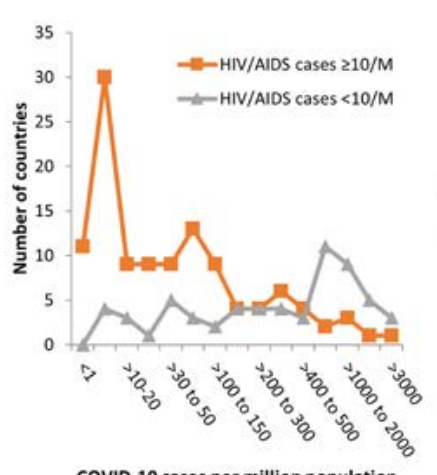

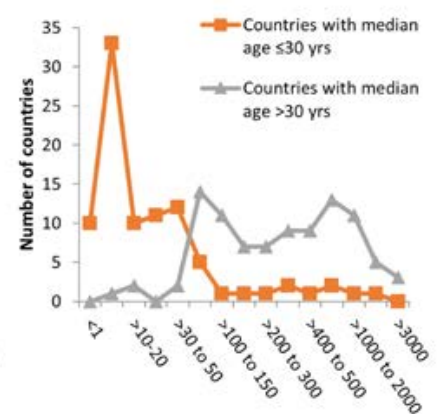

COVID-19 cases per million population

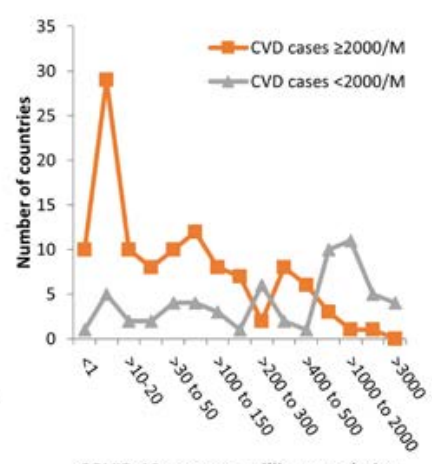

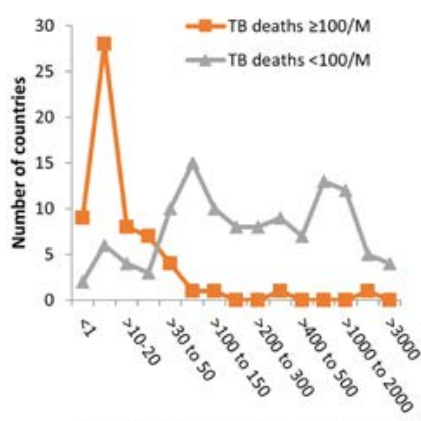

COVID-19 cases per million population

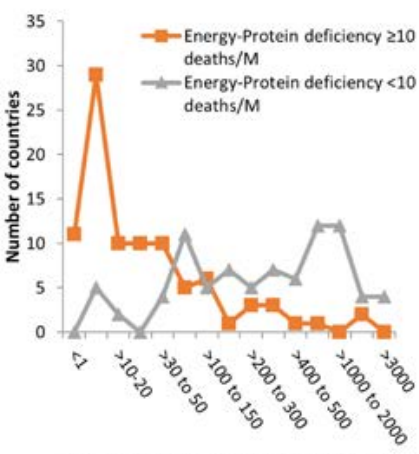

COVID-19 cases per million population

Fig. 2. Determinants of COVID-19 morbidity (per million population) patterns 
may be due to several factors involved in the spread of COVID-19, including national and international travel and social or religious congregations ${ }^{19,29}$. The similar instances of person-to-person contact and person-to-contaminated-surface contact in closed areas with virus-containing aerosols can result in Table 2. Odds ratios of epidemiological determinants with statistical significance for COVID-19 cases, deaths, and case fatality rates

\begin{tabular}{llll}
\hline Epidemiological Determinant Comparison & $\begin{array}{l}\text { Odds } \\
\text { Ratio }\end{array}$ & $\begin{array}{l}\text { Confidence } \\
\text { Interval }\end{array}$ & $\begin{array}{l}\text { Significance } \\
\text { Level }\end{array}$
\end{tabular}

Countries with non-mandatory versus mandatory BCG vaccination

$\begin{array}{lllll}1 & \text { Cases }>500 / M & 133 & 17.5-1011.37 & 0.01 \\ 2 & \text { Deaths }>20 / M & 42.96 & 9.62-191.85 & 0.01 \\ 3 & \text { CFR }>10 \% & 1.52 & 0.87-2.65 & >0.2\end{array}$

Countries with median age $>30$ years versus countries with median age $\leq 30$ years

$\begin{array}{lllll}1 & \text { Cases }>500 / M & 11.2 & 2.68-47.9 & 0.01 \\ 2 & \text { Deaths }>20 / M & 8.18 & 1.57-42.49 & 0.01 \\ 3 & \text { CFR }>10 \% & 0.73 & 0.50-1.08 & >0.2\end{array}$

Countries with tuberculosis deaths $<100 / \mathrm{M}$ (low TB burden) versus $\geq 100 / \mathrm{M}$ (high TB burden)

$\begin{array}{lllll}1 & \text { Cases }>500 / M & 24.46 & 1.72-347.80 & 0.01 \\ 2 & \text { Deaths }>20 / M & 10.83 & 1.57-92.2 & 0.05 \\ 3 & \text { CFR }>10 \% & 1.18 & 0.77-1.81 & >0.2\end{array}$

Countries with HIV/AIDS cases $<10 / \mathrm{M}$ versus countries with HIV/AIDS cases $\geq 10 / \mathrm{M}$

$\begin{array}{lllll}1 & \text { Cases }>500 / M & 13.09 & 3.92-43.68 & 0.01 \\ 2 & \text { Deaths }>20 / M & 4.75 & 1.43-15.76 & 0.01 \\ 3 & \text { CFR }>10 \% & 0.53 & 0.31-0.90 & 0.1\end{array}$

Countries with CVD cases $<200 /$ M versus $\geq$ Countries with $2000 / M$ CVD cases

$\begin{array}{lllll}1 & \text { Cases }>500 / M & 21.29 & 5.51-82.31 & 0.01 \\ 2 & \text { Deaths }>20 / M & 14.04 & 3.15-62.48 & 0.01 \\ 3 & \text { CFR }>10 \% & 0.76 & 0.51-1.15 & >0.2\end{array}$

Countries with energy-protein deficiency $<10$ deaths/M versus countries with $\geq 10$

$\begin{array}{lllll}1 & \text { Cases }>500 / M & 18.26 & 3.61-92.40 & 0.01 \\ 2 & \text { Deaths }>20 / M & 5.65 & 1.29-24.75 & 0.01 \\ 3 & \text { CFR }>10 \% & 0.5 & 0.26-0.93 & 0.05\end{array}$

Optional versus mandatory BCG vaccination in upper-middle-income countries

\begin{tabular}{|c|c|c|c|c|}
\hline 1 & Cases $>500 / \mathrm{M}$ & CNC & $\mathrm{CNC}$ & CNC \\
\hline 2 & Deaths $>20 / \mathrm{M}$ & CNC & $\mathrm{CNC}$ & $5.51 \mathrm{E}-09 *$ \\
\hline & CFR $>10 \%$ & CNS & $\mathrm{CNC}$ & $0.322629 *$ \\
\hline \multicolumn{5}{|c|}{ Optional versus mandatory BCG vaccination in high-income countries } \\
\hline 1 & Cases $>500 / \mathrm{M}$ & 134.62 & $8.66-2093.23$ & 0.01 \\
\hline & Deaths $>20 / M$ & 12.4 & 3.14-48.9 & 0.01 \\
\hline & CFR $>10 \%$ & 1.54 & $0.85-2.79$ & 0.2 \\
\hline \multicolumn{5}{|c|}{ Optional versus mandatory BCG vaccination in countries with median age $30-40$ years } \\
\hline 1 & Cases $>500 / \mathrm{M}$ & 46.8 & $2.23-982.58$ & 0.01 \\
\hline & Deaths $>20 / \mathrm{M}$ & 15.11 & $1.23-185.84$ & 0.01 \\
\hline & CFR $>10 \%$ & 4.33 & $1.19-15.78$ & 0.15 \\
\hline \multicolumn{5}{|c|}{ Optional versus mandatory BCG vaccination in countries with median age $>40$ years } \\
\hline & Cases $>500 / \mathrm{M}$ & CNC & $\mathrm{CNC}$ & CNC \\
\hline & Deaths $>20 / M$ & 36.67 & 2.87-467.98 & 0.01 \\
\hline & CFR $>10 \%$ & 1.3 & $0.54-3.10$ & 0.2 \\
\hline
\end{tabular}

CNC, cannot be calculated; * $\chi^{2}$ test statistics; BCG, Bacille-Calmette-Guérin vaccine; CFR, case fatality rate; M, million people; CVD, cardiovascular disease

Journal of Pure and Applied Microbiology 
$0.22, p=0.01$ ) with the number of people tested and the number of people testing positive (per million) for infection $(r=0.21, p=0.01)$. However, there was no correlation between literacy and mortality or CFR, probably because novel viruses like SARS-CoV-2 are not likely to discriminate between literate and illiterate people, as they all may be at the same plane of immunity in a given country.

Role of per capita GDP, per capita health expenditure, availability of safe water, food sufficiency, and sanitation in COVID-19

In this study, countries were classified into either high-, low-, or medium-income groups ${ }^{30}$ based on per capita GDP (using PPP statistics), to evaluate the effect of per capita GDP on COVID-19related morbidity and mortality. High PPP showed a strong positive correlation with morbidity $(r=$ $0.47 ; p<0.0001)$ and mortality $(r=0.25, p=0.01$; Table 1). Most wealthy countries with a high PPP have populations with a comparatively higher median age $\mathrm{e}^{8,12}$ and most have also discontinued the use of BCG $^{10,11}$. However, correlating and making conclusions regarding these factors independently may introduce bias. When comparing the countries with a high PPP $(>\$ 12,476)$, with and without a mandatory BCG vaccination policy, BCG vaccination showed a strong $(p<0.001)$

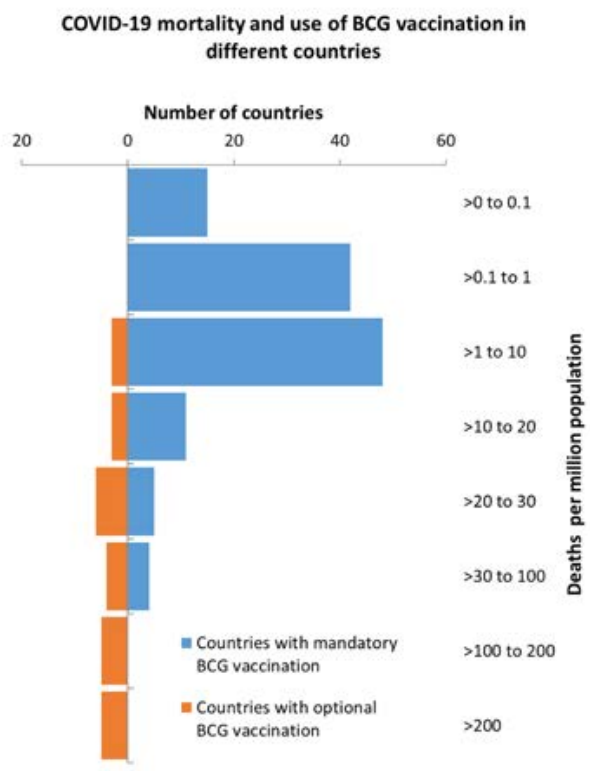

negative correlation with COVID-19 morbidity and mortality. In high-income countries with an optional BCG vaccination policy, the odds of having $>500$ COVID-19 cases per million people was 134.6 times higher $(99 \% \mathrm{Cl}, 8.66-2093.23)$ and the odds of having > 50 COVID-19 deaths per million people was 12.4 times higher $(99 \% \mathrm{Cl}, 3.14-48.9)$ than in high-income countries with a mandatory BCG vaccination policy. A similar correlation was evident between per capita health expenditure and COVID-19 morbidity $(r=0.47, \mathrm{p}<0.0001)$ and mortality $(r=0.25, p=0.01)$. However, the number of deaths owing to EPD was negatively correlated with COVID-19 morbidity $(r=0.19, \mathrm{p}<0.05)$ and mortality $(r=0.17, p=0.05)$. This indicated that the countries with insufficient resources may be failing to do the required number of tests to diagnose silent COVID-19 infections or alternatively, they may be protected because of the effect of mandatory BCG vaccination in all such countries because of their higher burden of $\mathrm{TB}^{12,15,16}$.

Is COVID-19 misdiagnosed?

Our analysis revealed that increased testing (number of tests per million people) was correlated with a lower COVID-19 CFR $(r=-0.17, p$ $=0.05)$, but higher morbidity $(r=0.57, p=0.001)$ and mortality $(r=0.19, p=0.05)$ rates, suggesting that COVID-19 might be over-diagnosed. The

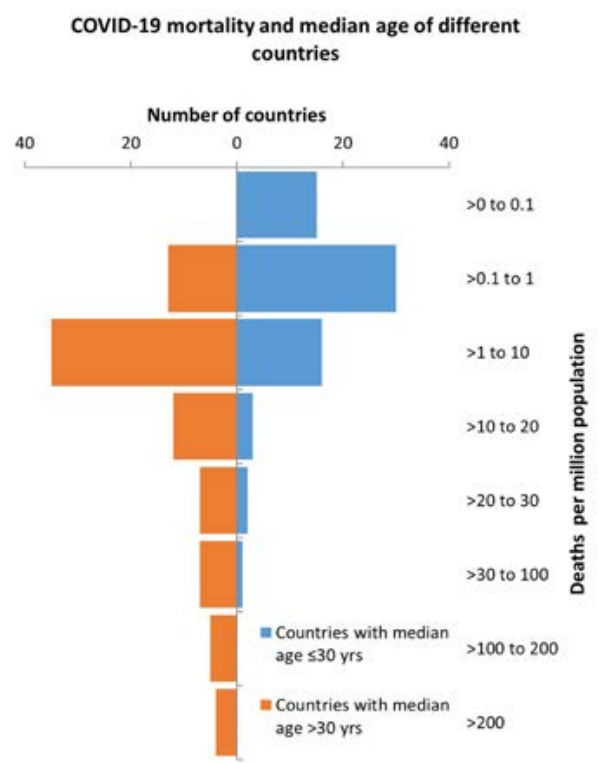

Fig. 3. Effect of mandatory BCG vaccination and median age of nations on COVID-19 mortality (per million population). 
present diagnosis is expected to overestimate SARS-CoV-2 infectivity and COVID-19 morbidity and mortality; since it is targeted to detect SARSCoV-2 in all suspected flu cases and all deaths in the pandemic regions. A higher number of cases have been registered in countries with high literacy rates and stronger economies, which also have higher testing capacity, further indicating that it may have been over-diagnosed.

Presently, testing for COVID-9 is aimed at identifying cases, without any goal of disease mapping and thus, until testing is performed with a sound sampling plan, the real incidence and prevalence of the disease cannot be accurately determined. Moreover, in the absence of real mapping of the disease, its determinants may not be identified correctly ${ }^{31}$.

Further, the number of individuals that tested positive for COVID-19 may be dissuasive in understanding the disease, owing to compromised positive and negative predictive values ${ }^{32,33}$. Falsepositivity in COVID-19 testing ${ }^{32}$ may be proving costly, but it can be tolerated for effective epidemic control $^{19}$. However, the current RT-PCR-based test has a high negative predictive value ${ }^{33}$ and may result in missing a large number of positive cases, which may be contributing to the propagation of the ongoing pandemic. Negative RT-PCR results for COVID-19 necessitate repeated testing to confirm the negative result. Thus, a better test with higher positive and negative predictive values is required to determine the true number of positive and negative cases. The requirement for repeated and parallel testing may force many poverty-ridden countries to completely abandon testing owing to its high cost. It becomes difficult to eradicate a disease when the necessities of life are becoming more difficult to maintain, as in most of lower-middle-income countries, because of high economical cost of testing, quarantine, and isolation of COVID-19 patients. A recent study on the prevalence of anti-SARS-CoV-2 antibodies in Santa Clara County ${ }^{34}$ revealed that the RT-PCRbased test can diagnose only a small fraction (1/50 to $1 / 80$ ) of SARS-CoV-2-infected people, indicating that the real infection fatality rate (IFR) may be just $0.1-0.9 \%$. The IFR for Santa Clara County was found to be $0.1-0.2 \%$, which represents one death in 480-820 infections. These observations indicate that there is much more research required to reach valid conclusions regarding the optimal strategy to map and control COVID-19.

COVID-19 mainly spreads through person-to-person contact during official and unofficial meetings, travel via public transport, and social and religious congregations. The contagiousness of COVID-19 has transformed it into a pandemic within a very short period

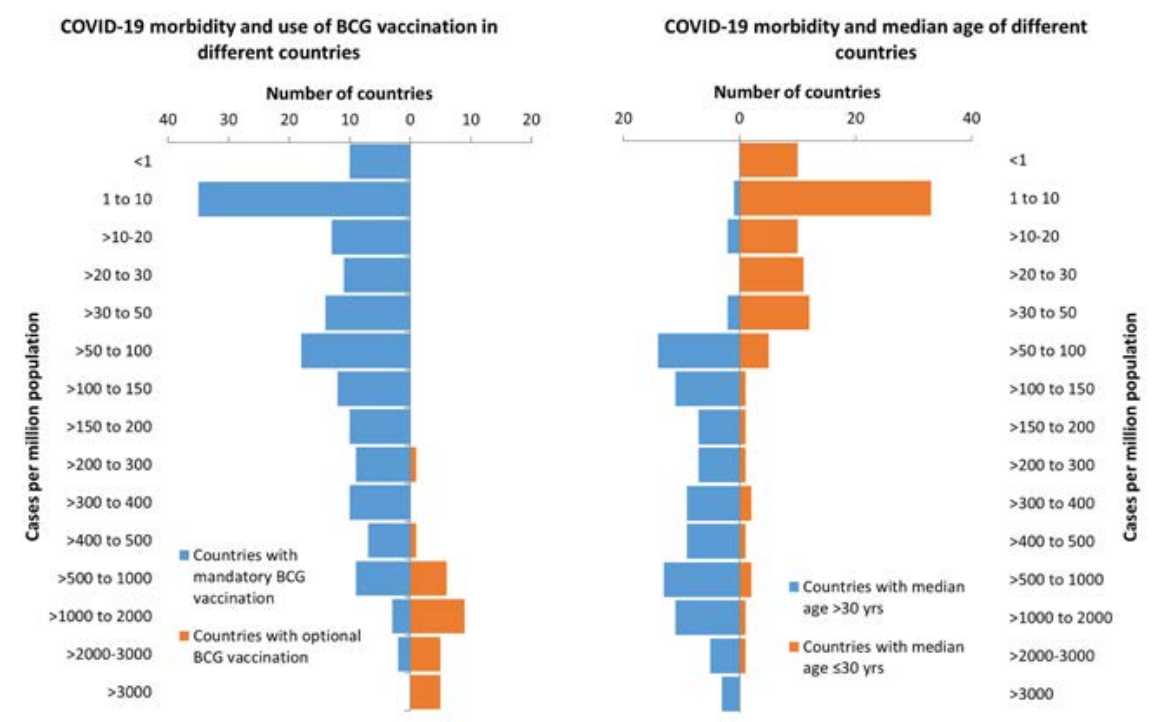

Fig. 4. Effect of mandatory BCG vaccination and median age of nations on COVID-19 morbidity (per million population). 
of 2 months. Although social distancing and lockdown are considered effective strategies to contain the disease and are in place around the world, many of the affected countries may fail to contain the pandemic. This failure may be owing to frequent violation ${ }^{29}$ and misunderstanding of the practice of social distancing and the fact that these are impracticable and costly means to control an epidemic caused by agents with high contagiousness $(R>2)$ and low morbidity and mortality rates ${ }^{2,3}$. At present, in most of the worst-affected countries, the COVID-19 morbidity rate is $1 \%$ and the mortality rate is $0.1 \%$, making it a fit case for an upcoming endemic. Eventually, to prevent further economic downturn, the lockdown will be lifted, which will allow people to interact freely and propagate the disease at low levels. Although the WHO has denied any beneficial correlation between BCG vaccination andCOVID-19 morbidity and mortality, the current analysis has revealed the possible utility of the BCG vaccine in reducing COVID-19 morbidity and mortality rates and the higher susceptibility of aged people to COVID-19.

\section{ACKNOWLEDGMENTS}

All the listed author(s) are thankful to their representative universities/institutes for providing the related support to compile this work.

\section{CONFLICT OF INTEREST}

The authors declares that there is no conflict of interest.

\section{AUTHORS' CONTRIBUTION}

All the listed author(s) have made a substantial, direct and intellectual contribution to the work, and approved it for publication.

\section{FUNDING}

None.

\section{ETHICS STATEMENT}

This article does not contain any studies with human participants or animals performed by any of the authors.

\section{AVAILABILITY OF DATA \\ Not applicable.}

\section{REFERENCES}

1. COVID-19 Coronavirus pandemic. https://www. worldometers.info/coronavirus/ (2020).

2. Maharaj S, Kleczkowski A. Controlling epidemic spread by social distancing: Do it well or not at all. BMC Public Health. 2012;12:679.

3. Fast SM, Gonzalez MC, Markuzon N. Costeffective control of infectious disease outbreaks accounting for societal reaction. PLOS ONE. 2015;10:e0136059.

4. World Health Organization. Report of the WHOChina Joint Mission on Coronavirus Disease 2019 (COVID-19), 16-24 February 2020. https://www. who.int/docs/default-source/coronaviruse/ who-china-joint-mission-onCOVID-19-finalreport.pdf

5. Can pets carry COVID-19 virus? Should we have simultaneous quarantine of pets from owners testing positive for COVID-19? 24 March 2020. https://azad-azadindia.blogspot.com/2020/03/ can-pets-carry-COVID-19-virus-should-we.html 2020.

6. Coronavirus (COVID-19). https://www.cdc.gov/ coronavirus/2019-ncov/index.html 2020

7. These lab animals will fight Coronavirus. https:// www.nytimes.com/2020/03/14/science/ animals-coronavirus-vaccine.html 2020.

8. Worldometer. 1 April 2020. https://www. worldometers.info/ 2020.

9. List of countries by GDP (PPP). https:// en.wikipedia.org/wiki/List_of_countries_by_ GDP_(PPP) 2020.

10. Hall $M$. What is purchasing power parity (PPP). www.investopedia.com/updates/purchasingpower-paity-ppp/ 2020.

11. Zwerling A, Behr MA, Verma $A$, et al. The BCG World Atlas: A Database of Global BCG Vaccination Policies and Practices. PLOS Medicine. 2011;8(3):e1001012. https://doi. org/10.1371/journal.pmed.1001012.

12. BCG vaccine. https://en.wikipedia.org/wiki/ BCG_vaccine 2020 .

13. Countries by Median Age 2018. https:// worldpopulationreview.com/countries/ 2018.

14. Health Data. http://ghdx.healthdata.org/gbdresults-tool. 2020.

15. World health statistics 2019: monitoring health for the SDGs, sustainable development goals. Geneva: World Health Organization. Licence: CC BY-NC-SA 3.0 IGO 2019.

16. TB Facts.org. https://tbfacts.org/high-burdentb/ 2020.

17. Iqbal NT, Hussain R. Non-specific immunity of BCG vaccine: A perspective of BCG immunotherapy. Trials Vaccinol. 2014;3:143-149. 
18. Covian C, Fernandez-Fierro A, RetamalDiaz $A$, et al. BCG-induced cross-protection and development of trained immunity: implication for vaccine design. Frontiers in Immunology. 2019;29:2806. doi: 10.3389/ fimmu.2019.02806. eCollection 2019.

19. Singh BR, Gandharva R. Are BCG Vaccination, Population Density, Median Age and Poverty Important Determinants of COVID-19 Pandemic Spread, Morbidity and Mortality? DOI: 10.13140/ RG.2.2.21116.49282. 2020. https://www. researchgate.net/publication/340443017_Are_ BCG_Vaccination_Population_Density_Median_ Age_and_Poverty_Important_Determinants_ of_COVID-19_Pandemic_Spread_Morbidity_ and_Mortality 2020.

20. Miller A, Reandelar MJ, Fasciglione K, Roumenova V, Li Y, Otazu GH. Correlation between universal BCG vaccination policy and reduced morbidity and mortality for COVID-19: an epidemiological study.

21. Bacille Calmette-Guerin (BCG) vaccination and COVID-19. World Health Organization Scientific brief 12 April 2020. https://www.who.int/newsroom/commentaries/detail/bacille-calmettegu\%C3\%A9rin-(bcg)-vaccination-and-COVID-19.

22. Reducing Health Care Workers Absenteeism in COVID-19 Pandemic Through BCG Vaccine (BCGCORONA). https://clinicaltrials.gov/ct2/show/ NCT04328441.

23. BCG Vaccination to Protect Healthcare Workers Against COVID-19 (BRACE). https://clinicaltrials. gov/ct2/show/NCT04327206.

24. Guan W, Liang W, Zhao Y, et al. Comorbidity and its impact on 1590 patients with COVID-19 in China: A Nationwide Analysis. Eur Respir. 2020; 2000547. DOI: 10.1183/13993003.00547-2020.

25. Age, Sex, Existing Conditions of COVID-19 Cases and Deaths. https://www.worldometers. info/coronavirus/coronavirus-age-sexdemographics/ 2020.
26. The Epidemiological Characteristics of an Outbreak of 2019 Novel Coronavirus Diseases (COVID-19) - China CCDC, February 172020.

27. Report of the WHO-China Joint Mission on Coronavirus Disease 2019 (COVID-19) [Pdf] World Health Organization, Feb. 28, 2020.

28. Tarwarter P, Martin C. Effects of population density on the spread of disease. Complexity. 2001;6:2936.

29. Tabligi Jamat Case Story behind the COVID hotspot that set cops on a frantic nationwide hunt. 2 April 2020. The Economic Times. https:// economictimes.indiatimes.com/news/politicsand-nation/tablighi-jamaat-case-story-behindthe-COVID-hotspot-that-set-cops-on-a-franticnationwide-hunt/articleshow/74947692.cms 2020.

30. Country classification. https://www.un.org/ en/development/desa/policy/wesp/wesp_ current/2014wesp_country_classification.pdf 2020.

31. Silver N. Coronavirus case counts are meaningless. FiveThirtyEight 4 April 2020. https://fivethirtyeight.com/features/ coronavirus-case-counts-are-meaningless/ 2020.

32. Zhuang GH, Shen MW, Zeng LX, et al. Potential false-positive rate among the 'asymptomatic infected individuals' in close contacts of COVID-19 patients. Pubmed. 2020;41:485-488. doi:10.3760/cma.j.cn112338-20200221-00144.

33. Sturts A. Comparing RT-PCR and chest ct for diagnosing COVID-19. HCPLive 17 March 2020. https://www.mdmag.com/medical-news/ comparing-rt-pcr-and-chest-ct-for-diagnosingCOVID19 2020.

34. Bendavid E, Mulaney B, Sood N, Shah S, Ling E, Bromley-Dulfano R, et al. COVID-19 Antibody Seroprevalence in Santa Clara County, California. medRxiv. 2020.04.14.20062463. doi: https://doi. org/10.1101/2020.04.14.20062463. 